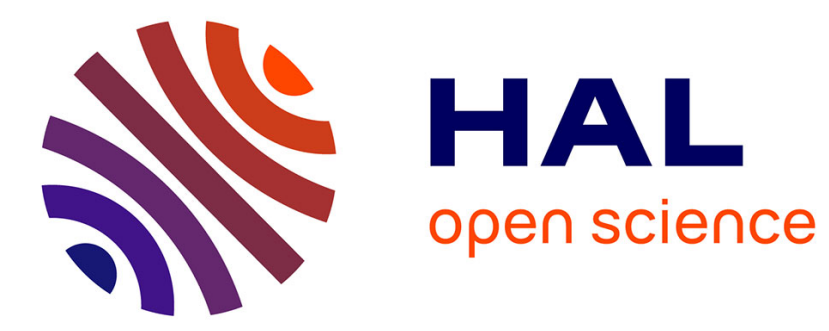

\title{
Prostate segmentation on T2 MRI using Optimal Surface Detection
}

Ke Wu, Carole Garnier, Huazhong Shu, Jean-Louis Dillenseger

\section{To cite this version:}

Ke Wu, Carole Garnier, Huazhong Shu, Jean-Louis Dillenseger. Prostate segmentation on T2 MRI using Optimal Surface Detection. Innovation and Research in BioMedical engineering, 2013, 34 (4-5), pp.287-290. 10.1016/j.irbm.2013.07.005 . inserm-00879165

\section{HAL Id: inserm-00879165 https://www.hal.inserm.fr/inserm-00879165}

Submitted on 1 Nov 2013

HAL is a multi-disciplinary open access archive for the deposit and dissemination of scientific research documents, whether they are published or not. The documents may come from teaching and research institutions in France or abroad, or from public or private research centers.
L'archive ouverte pluridisciplinaire HAL, est destinée au dépôt et à la diffusion de documents scientifiques de niveau recherche, publiés ou non, émanant des établissements d'enseignement et de recherche français ou étrangers, des laboratoires publics ou privés. 


\title{
Prostate segmentation on T2 MRI using Optimal Surface Detection
}

\author{
Wu Ke $\mathrm{K}^{\mathrm{a}, \mathrm{b}, \mathrm{c}}$, Garnier Carole ${ }^{\mathrm{a}}$, Shu Huazhong ${ }^{\mathrm{b}, \mathrm{c}}$, Dillenseger Jean-Louis ${ }^{\mathrm{a}, \mathrm{c}}$, \\ ${ }^{a}$ Inserm, U1099, Rennes, F-35000, France ; Université de Rennes 1, LTSI, Rennes, \\ F-35000, France \\ ${ }^{b}$ Laboratory of Image Science and Technology, Department of Computer Science and \\ Engineering, Southeast University, 210096, Nanjing, China \\ ${ }^{c}$ CRIBS, Laboratoire International Associé, Inserm, Université de Rennes 1, France, \\ Southeast University, Nanjing, China
}

\begin{abstract}
This paper deals with a T2 MRI prostate segmentation method. We assume to have an initial surface mesh obtained interactively or after a first rough segmentation. The surface of the prostate is then searched within the initial mesh neighborhood using the Optimal Surface Detection algorithm (OSD). This algorithm is based on the construction of a directed graph from the information obtained around the initial mesh. The optimal surface is then obtained by a graph cut. Three different cost functions for the graph have been explored, one based on the local gradient, another on a statistical model of shape and a third on a model of gradient profile. The parameters of this method have been tuned on 33 different T2 MRI volumes.
\end{abstract}

Keywords: Prostate, surface segmentation, T2 MRI

\section{Introduction}

The segmentation of the prostate surface in T2 MRI is a difficult task because several prostatic zones are not homogeneous and its surface has got little information (hyposignal of the capsule) with gray level values close to those of the neighboring organs surface (bladder and rectum). In this paper we will adapt a method previously developed for the semiautomated prostate segmentation on transrectal ultrasound images to the T2 MRI case [1]. This method uses the Optimal Surface Detection (OSD) [2] to refine the prostate 
surface segmentation in the neighborhood of a first approximate surface obtained interactively or after a first rough segmentation. This paper will deal with the adaptation and the tuning of the OSD in the T2 MRI context. Two attempts to introduce statistical shape information will also be presented.

\section{Material et methods}

\subsection{Optimal Surface Detection - OSD}

As an initial stage we assume to have a first approximative prostate surface mesh. This initial mesh can be defined semi-automatically (e.g. an ellipsoid adjusted on 6 initial points on the boundary of the prostate volume -apex, base and 4 points within the central axial plane- defined interactively by a radiologist [1]) or after a first more automated rough segmentation.

The principle of the OSD algorithm is to consider the segmentation problem as the computation of a minimum s-t cut in an oriented weighted graph [2]. The graph is built as follows. Each vertex $i$ of the initial mesh serves as a graph node. From each vertex $i$ a column of nodes is created along the surface normal $\mathbf{N}_{i}$ and distributed on both side of the surface (Fig.1). The search distances inside and outside the surface are set respectively to In $D_{i}$ and $O u t D_{i}$. Two auxiliary nodes $s$ and $t$ (source and sink respectively in Boykov's terminology [3]) will allow the volume segmentation into two classes "inside" and "outside" of the surface.

These nodes are connected by links. The weights of the links will be used to encode different information (local image-based surface energy, shape constraints, gradient profile, ...). Several kind of links will be defined:

1) Intra-column links of infinite weights connect together two adjacent nodes of a same column from outside to inside. This construction insures that the surface intersects each column on only one of its nodes.

2) Inter-columns links of infinite weights will be used as a regularity constraint. One node $N_{i, c}$ of column $i$ and position $c$ on the column is connected to the node $N_{j, \max \left(0, c-\Delta_{i, j}\right)}$ of column $j$, with $\Delta_{i, j}$ a smoothness constraint (Fig.1). The purpose of this constraint is to ensure that a surface can only move from distance less than $\Delta$ from one column to another. $\Delta$ will be adapted according to the distance between two neighbor vertexes $p_{i}$ and $p_{j}$ by $\Delta_{i, j}=\left\lfloor\delta\left\|p_{i}-p_{j}\right\|\right\rfloor$ with $\delta$ a constant.

3) The surface local energy is encoded as follows. In a first step a cost function $C$ based on local image features and/or shape probability and/or 
some gradient profile model is defined. This function is then used to assign a weight $w_{i, c}$ to each node (with $c=0$ the lowest node in a column):

$$
w_{i, c}= \begin{cases}C_{i, c}, & \text { if } c=0 \\ C_{i, c}-C_{i, c-1}, & \text { otherwise }\end{cases}
$$

The nodes with negative weights will be linked to the source $s$ with a weight $\left|w_{i, c}\right|$ and the nodes with positive (or null) weights to the sink $t$ with a weight $w_{i, c}$.

4) The optimal surface is finally obtained with a min-cut/max-flow graph cut algorithm [3].

\subsection{Cost function}

The definition of the cost function is the key point of the OSD algorithm. Three different classes of cost functions have been tested based on: 1) local image information (gradients), 2) a shape probability function and 3) a gradient profile model.

Image-based feature: gradients. The image-based surface energy is based on the smoothed gradient of the volume $V: E(x, y, z)=\left\|\nabla\left(G_{\sigma} * V(x, y, z)\right)\right\|=$ $\|\mathbf{G}(x, y, z)\|$ with $G_{\sigma}$ a Gaussian filter with standard deviation $\sigma$. We tested 3 different cost functions: POS_GDT promoting positive gradients (low to high value transitions which means the same direction as the surface normal):

$C_{i, c}=-\left\|\mathbf{G}\left(x_{i, c}, y_{i, c}, z_{i, c}\right)\right\| \frac{s_{i, c}+1}{2}$

with $s_{i, c}=\frac{\mathbf{G}\left(x_{i, c}, y_{i, c}, z_{i, c}\right) \cdot \mathbf{N}_{i}}{\left\|\mathbf{G}\left(x_{i, c}, y_{i, c}, z_{i, c}\right)\right\|}$.

EG_GDT promoting negative gradients:

$C_{i, c}=-\left\|\mathbf{G}\left(x_{i, c}, y_{i, c}, z_{i, c}\right)\right\| \frac{\left|s_{i, c}-1\right|}{2}$.

ALL_GDT promoting any directions:

$C_{i, c}=-\left\|\mathbf{G}\left(x_{i, c}, y_{i, c}, z_{i, c}\right)\right\|$.

Shape probability function. A shape model is first constructed from a training set of prostate contours manually extracted by an urologist on a set of prostate volumes (see $\S 3.1$ for the data): 1) For each volume, a surface is built from the contours. For this, a local prostate-based reference system is first defined (the first axis is aligned from the apex to the base, the reference origin is set as the middle of the apex-base segment, the 2 other axes are adjusted to the prostate surface). Then the expert surface is regularly resampled within this local reference system in order to form a regular mesh. 2) The meshes of all the surfaces of the training set are aligned using the generalized Procustes algorithm [4] implemented in VTK. This algorithm gives 
on one hand an average shape and on the other all the forms aligned on the average shape. A probability map is then estimated from the aligned shapes: a) a 3D distance map is build on each shape; b) the distance map is converted into a Gaussian distance map: $\exp \left(-\frac{d^{2}}{2 \sigma_{m}^{2}}\right)$ with $\sigma_{m}$ a constant; and c) the probability map is deduced by averaging the Gaussian maps of all the aligned shapes. For the segmentation of a new prostate the probability map must be adjusted on the data. The model average shape is aligned onto the initial mesh of the data to segment. The estimated rigid transformation matrix (translation, rotation scaling) is finally applied on the probability map. The node cost value is the gradient-based cost multiplied by the probability at the node voxel position. Cost functions that add the probability to the gradient-based cost were also tested. As they lead to worse performance, results were not reported.

Gradient profile model. The idea is to build a statistical model of the gradient profiles variation from a learning set and then use this model to assign some costs [5]. Surfaces of the training set are first aligned as described previously. At this stage, we have $K$ shapes each sampled on $N$ vertexes. $N$ gradient profile models are then built as described in [5]. A gradient profile $g_{i j}$ is estimated at each sample $i(i=1, \ldots, N)$ in each training surface $j(j=$ $1, \ldots, K)$. Each profile consists in the energy of 10 voxels on both sides of the surface. For each $i$ we have a profile vector $\mathbf{g}_{\mathbf{i}}$ which can be normalized as: $\mathbf{g}_{\mathbf{i}} \rightarrow \frac{1}{\sum_{j}\left|g_{i j}\right|} \mathbf{g}_{\mathbf{i}}$. If we assume that all profiles are distributed as a multivariate Gaussian, the appearance on one sample can be modeled by the mean profile $\hat{g}_{i}$ and the covariance matrix $\mathbf{S}_{\mathbf{g i}}$. The quality of the fit of a new profile $g_{s i}$ to the model can be estimated by the Mahalanobis distance: $f\left(g_{s i}\right)=\left(g_{s i}-\right.$ $\left.\hat{g}_{i}\right)^{T} \mathbf{S}_{\mathbf{g i}}{ }^{-1}\left(g_{s i}-\hat{g}_{i}\right)$. For the segmentation of a new prostate, the initial mesh must be aligned on its local prostate based reference system and resampled in order to ensure correspondence to the model vertices. Gradients profiles $g_{i, c}$ are computed for each voxel that corresponds to a node $N(i, c)$ in the graph. The node cost value is then defined as: $C_{i, c}=f\left(g_{i, c}\right)-\max \left(f\left(g_{s}\right)\right)$ where $\max \left(f\left(g_{s}\right)\right)$ is the maximum Mahalanobis distance over all the computed profiles in the current image. 


\section{Results}

\subsection{Data and evaluation protocol}

The algorithms were tested on $33 \mathrm{~T} 2$ axial MRI volumes from 5 different devices. We had images of different qualities and image size ranged from $256 \times 256 \times 24$ to $720 \times 720 \times 20$ voxels with pixel size ranging from 0.391 to $0.781 \mathrm{~mm}$ and axial slice thicknesses from 2.63 to $4.50 \mathrm{~mm}$. On each of the 33 volumes, an urologist delineates manually the contours of the prostate. These contours will serve as ground truth or to build the shape and the gradient profile model. The MRI 3D images were interpolated along $z$ before segmenting the prostate.

The performance of a specific segmentation scheme is evaluated with the Volume Overlap metric: $\mathrm{VO}=\frac{V_{M} \cap V_{A}}{V_{M} \cup V_{A}}$ with $V_{M}$ and $V_{A}$ respectively the expert (manual) and the OSD (automatic) segmented volume. The mean in $\%$ and the standard deviation (std) of all the 33 VOs serve as global performance metrics. However, it has to be noticed that this evaluation scheme is just one indicator of a performance. Only one manual segmentation per volume from one expert has been performed. Other studies with several segmentations per volume from one or several experts showed relative huge intra- and interobserver variabilities [6]. So the results of our VO metric should be used only with caution and should only be considered as a relative performance measurement which gives high performance increases in some cases (e.g. cases $8,11,25)$ but also really important performance decreases (e.g. case 10).

\subsection{Graph parameters setting}

The graph parameters setting is performed on the classical OSD algorithm with the gradient-based cost function. Five parameters have to be tuned: InD and OutD the search distances inside and outside the surface, $\delta$ from the hard shape constraint, $\sigma$ the Gaussian filter standard deviation and the promoted gradient direction (POS_GDT, NEG_GDT or ALL_GDT). We tuned sequentially $\delta, \sigma, \operatorname{In} D$ and $O u t D$ and the gradient direction. For a specific parameter, the value which gives the best performance is kept for the next parameter tuning. The optimal setting after our exhaustive search for the several parameters variations was: $\delta=1 / 2, \sigma=1 \mathrm{~mm}, \operatorname{In} D=-5 \mathrm{~mm}$, Out $D=15 \mathrm{~mm}$ and the ALL_GDT gradient direction. This setting gives a mean VO of $77.2 \%$ with a standard deviation of $5.5 \%$.

The VO of $77.2 \%$ could be considered as relatively low. However, it should be noticed that due to the high interslice spacing of T2 MRI, the 
prostate is generally described on only 6 or 7 slices and that it is relatively difficult even for the radiologist or urologist to define the prostate shape in the planes tangent to the organ near the base or the apex. We also showed in a previous study [1] that the $\mathrm{VO}$ obtained between the prostate extracted on 3D US images (which had a better interslice spacing as T2 MRI) by two different experts or twice by the same expert presented a mean VO value of around $83 \%$. Even if the score obtained on T2 MRI could not be compared to this obtained on the US volume, an analog behavior should be considered.

\subsection{Shape probability and gradient profile model}

In order to evaluate these algorithms, the training set used for the segmentation of a specific prostate volume was built from the expert manual segmentations of all the other 32 volumes. We first tuned the shape probability cost function based method on 7 volumes in order to find the optimal $\sigma_{m}$ parameter $\left(\sigma_{m}=8\right)$.

Fig. 2 compares the VO obtained by the gradient based, shape probability and gradient profile model for all the 33 cases. The shape probability increases slightly the overlap in some cases but globally gives less performing results. A same conclusion can be drawn with the gradient profile modelbased cost function which gives some high performance increasing in some cases (e.g. cases $8,11,25)$ but also some really performance decreases (e.g. case 10).

Some of the assumptions like a Gaussian distribution of the profiles are not always pertinent. Also because T2 MRIs of diseased prostates have a high variability in texture the gradient search (or model) has difficulty to separate the prostate boundary from its surroundings or its inside part.

\section{Conclusion}

The shape probability and gradient profile model can in some cases increase the performance of the segmentation but also can lead to very poor results. Such algorithms can be interesting in a segmentation scheme where the end-user (the urologist) can choose the best results in his sense between the several intermediary results obtained using the different cost functions. This work is part of the French MULTIP project supported by an ANR Grant (ANR-09-TECS-011-06). 


\section{References}

[1] C. Garnier, J.-J. Bellanger, K. Wu, H. Shu, N. Costet, R. Mathieu, R. de Crevoisier, J.-L. Coatrieux, Prostate segmentation in HIFU therapy, IEEE Trans. Med. Imag. 30 (3) (2011) 792-803.

[2] K. Li, X. Wu, D. Chen, M. Sonka, Efficient optimal surface detection: theory, implementation, and experimental validation, in: Proc. SPIE Medical Imaging, Vol. 5370, San Diego, 2004, pp. 620-627.

[3] Y. Boykov, V. Kolmogorov, An experimental comparison of mincut/max- flow algorithms for energy minimization in vision, IEEE Trans. Pattern Anal. Mach. Intell. 26 (9) (2004) 1124-1137.

[4] J. Gower, Generalized Procrustes analysis, Psychometrika 40 (1975) 3351.

[5] T. F. Cootes, C. J. Taylor, Statistical models of appearance for medical image analysis and computer vision, in: Proc. SPIE Medical Imaging, Vol. 4322, San Diego, 2001, pp. 236-248.

[6] C. Fiorino, M. Reni, A. Bolognesi, G. M. Cattaneo, R. Calandrino, Intraand inter-observer variability in contouring prostate and seminal vesicles: implications for conformal treatment planning., Radiother Oncol 47 (3) (1998) 285-292. 


\section{List of Figures}

1 Graph construction. . . . . . . . . . . . . . . . . 9

2 Comparison of local gradient-, shape probability- and gradient profile model-based cost functions performance. Volume overlap metric for all the 33 volumes. . . . . . . . . . . . . . 9 


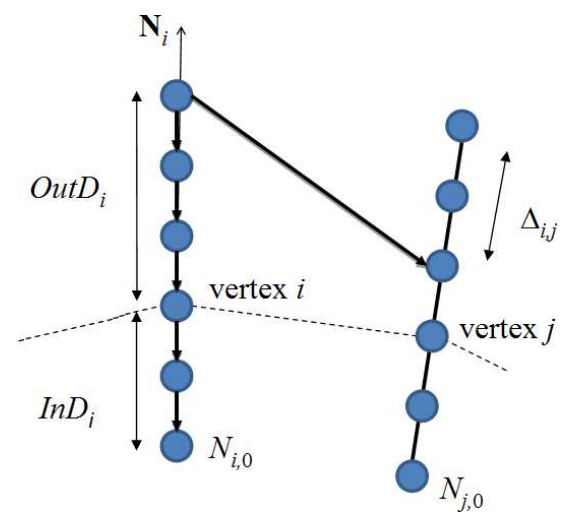

Figure 1: Graph construction.

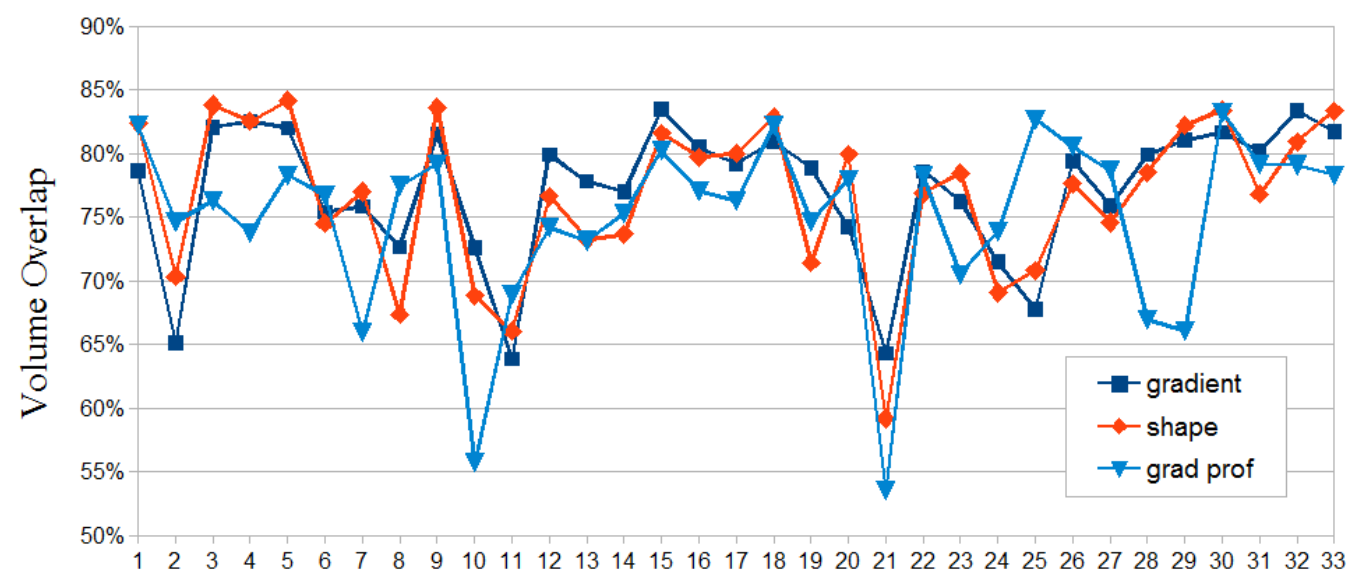

Figure 2: Comparison of local gradient-, shape probability- and gradient profile modelbased cost functions performance. Volume overlap metric for all the 33 volumes. 